\title{
A Fast and Efficient Numerical Algorithm for the Nonlocal Conservative Swift-Hohenberg Equation
}

\author{
Jingying Wang ${ }^{1}$ and Shuying Zhai ${ }^{1,2}$ \\ ${ }^{1}$ Fujian Province University Key Laboratory of Computation Science, School of Mathematical Sciences, \\ Huaqiao University, Quanzhou 362021, China \\ ${ }^{2}$ College of Mathematics and Systems Science, Xinjiang University, Urumqi 830046, China \\ Correspondence should be addressed to Shuying Zhai; syzmath@163.com
}

Received 4 August 2019; Revised 15 October 2019; Accepted 23 October 2019; Published 6 January 2020

Academic Editor: Ruben Sevilla

Copyright (c) 2020 Jingying Wang and Shuying Zhai. This is an open access article distributed under the Creative Commons Attribution License, which permits unrestricted use, distribution, and reproduction in any medium, provided the original work is properly cited.

\begin{abstract}
In this paper, we consider a new Swift-Hohenberg equation, where the total mass of this model is conserved through a nonlocal Lagrange multiplier. Based on the operator splitting method and spectral method, a fast and efficient numerical algorithm is proposed. Three numerical examples in both two and three dimensions are provided to illustrate that the proposed algorithm is a practical, accurate, and efficient simulation tool for the nonlocal Swift-Hohenberg equation.
\end{abstract}

\section{Introduction}

The Swift-Hohenberg model [1] was first introduced by Swift and Hohenberg in studies of Rayleigh-Bénard convection and has become one of the paradigms of the nonlinear dynamical system leading to complex pattern formation. Recent applications of the Swift-Hohenberg equation have been extensively spanned in complex fluids and biological tissues $[2,3]$. The Swift-Hohenberg model is derived by using the $L^{2}$-gradient flow of the Lyapunov energy functional [4]

$$
E(\phi)=\int_{\Omega}\left(\frac{\phi}{2}(\Delta+1)^{2} \phi+F(\phi)\right) \mathrm{d} \mathbf{x},
$$

where $\Omega$ is a bounded regular domain $[a, b]^{n}(n=1,2,3)$ and $\phi$ is the density field. $F(u)=(1 / 4) \phi^{4}-(\eta / 3) \phi^{3}-(\epsilon / 2) \phi^{2}$ is the nonlinear smoothing potential, and $\eta \geq 0$ and $\epsilon>0$ are constants with physical significance.

By taking the variational derivative of (1) in $L^{2}$ norm, the following Swift-Hohenberg equation is obtained:

$$
\phi_{t}=-\frac{\delta E(\phi)}{\delta \phi}=-\left((\Delta+1)^{2} \phi+f(\phi)\right), \quad(\mathbf{x}, t) \in \Omega \times(0, T],
$$

where $\delta E(\phi) / \delta \phi$ denotes the variational derivative and $f(u)=F^{\prime}(u)=\phi^{3}-\eta \phi^{2}-\epsilon \phi$. Thus, the total energy $E(\phi)$ is not increasing with time

$$
\frac{\mathrm{d}}{\mathrm{d} t} E(\phi)=\int_{\Omega} \frac{\delta E(\phi)}{\delta \phi} \frac{\partial \phi}{\partial t} \mathrm{~d} \mathbf{x}=-\int_{\Omega}\left(\frac{\partial \phi}{\partial t}\right)^{2} \mathrm{~d} \mathbf{x} \leq 0
$$

The Swift-Hohenberg equation (2) is a fourth-order nonlinear partial differential equation, and we cannot generally obtain the analytic solution for the arbitrary initial conditions. Therefore, computer simulations play an essential role in understanding the dynamical behavior. Till now, a large number of works have been proposed to study the Swift-Hohenberg equation [5-12].

Since the Swift-Hohenberg model (2) is derived by using the $L^{2}$-gradient flow, the total mass of the unknown variable is not conserved with time. However, in Elder's phase field crystal model, the phase field variable is interpreted as the density of atoms from the perspective of physical significance; thus, the total mass of the phase field variable is expected to be conserved, such as the CahnHilliard equation [13, 14]. Zhang and Yang [15] very recently proposed a conservative Swift-Hohenberg equation with a nonlocal Lagrange multiplier to cancel out the variation in mass without influencing the original energy 
law. The conservative Swift-Hohenberg equation is as follows:

$$
\begin{array}{r}
\phi_{t}+(\Delta+1)^{2} \phi+f(\phi)-\frac{1}{|\Omega|} \int_{\Omega}(\phi+f(\phi)) \mathrm{d} \mathbf{x}=0, \\
(\mathbf{x}, t) \in \Omega \times(0, T],
\end{array}
$$

subject to the initial condition

$$
\phi(\mathbf{x}, 0)=\phi_{0}(\mathbf{x}), \quad \mathbf{x} \in \bar{\Omega},
$$

The periodic boundary condition is adopted here since it is usually used to remove all boundary integrals (cf. [15]). Note that when $(1 /|\Omega|) \int_{\Omega}(\phi+f(\phi)) \mathrm{d} \mathbf{x}=0$, equation (4) degenerates back to the standard Swift-Hohenberg model (2).

The nonlocal term $(1 /|\Omega|) \int_{\Omega}(\phi+f(\phi)) \mathrm{d} \mathbf{x}$ can be understood as a nonlocal Lagrange multiplier for the mass constraint

$$
\frac{\mathrm{d}}{\mathrm{d} t} M(\phi)=\frac{\mathrm{d}}{\mathrm{d} t} \int_{\Omega} \phi(\mathbf{x}, t) \mathrm{d} \mathbf{x}=0
$$

Furthermore, system (4) still satisfies energy dissipative law (3). Taking the $L^{2}$ inner product of (4) with $\phi_{t}$, integrating by parts and using (6), we have

$$
\begin{aligned}
\frac{\mathrm{d}}{\mathrm{d} t} E(\phi)= & \int_{\Omega}\left(\phi(\Delta+1)^{2}+f(\phi)\right) \phi_{t} \mathrm{~d} \mathbf{x} \\
= & \int_{\Omega} \phi_{t}\left((\Delta+1)^{2} \phi+f(\phi)\right) \mathrm{d} \mathbf{x}-\frac{1}{|\Omega|} \int_{\Omega}(\phi \\
& +f(\phi)) \mathrm{d} \mathbf{x} \int_{\Omega} \phi_{t} \mathrm{~d} \mathbf{x} \\
= & -\int_{\Omega}\left(\phi_{t}\right)^{2} \mathrm{~d} \mathbf{x} \leq 0,
\end{aligned}
$$

where $E(\phi)$ is defined by (1).

Compared to a large number of studies for the standard Swift-Hohenberg equation (2), there are few numerical results on the conservative Swift-Hohenberg equation (4) because the extra nonlocal term brings up substantial numerical challenges to developing fast and efficient algorithms. Zhang and Yang [15] proposed a second-order, unconditionally energy stable scheme by combining the invariant energy quadratization (IEQ) approach with the stabilization technique. The law of conservation of mass of the proposed scheme was not verified in this article [15].

In this work, we aim to develop a fast explicit algorithm based on the operator splitting method $[14,16,17]$. The main idea of the method is to solve problem (4) through three parts: the linear homogeneous heat equation was first solved via the Fourier spectral method and then the nonlinear part and the nonlocal equation were solved explicitly.

The rest of this paper is organized as follows. In Section 2, a fast explicit operator splitting method is proposed for solving (4). In Section 3, we test the accuracy and applicability of the obtained numerical method in three examples including a 3D example. Finally, we summarize the present work and discuss the future work in Section 4.

\section{Time-Splitting Spectral Method}

In this section, we present a time-splitting spectral method to simulate the asymptotic behavior of the solution of equation (4). The proposed method is based on the operator splitting method for the time and spectral method for the space. For simplicity, we only consider two-dimensional (2D) space. The $1 \mathrm{D} / 3 \mathrm{D}$ case is defined analogously.

The operator splitting method for the conservative Swift-Hohenberg equation is constructed as follows. We rewrite equation (4) in the following abstract initial value problem:

$$
u_{t}=\mathscr{A} u+\mathscr{B} u+\mathscr{C} u
$$

where the operators $\mathscr{A}, \mathscr{B}$, and $\mathscr{C}$ are defined as

$$
\begin{aligned}
& \mathscr{A} \phi=-(\Delta+1)^{2} \phi, \\
& \mathscr{B} u=-f(\phi), \\
& \mathscr{C} u=\frac{1}{\Omega} \int_{\Omega}(\phi+f(\phi)) \mathrm{d} \mathbf{x} .
\end{aligned}
$$

Thus, we can evolve the solution from $t$ to $t+\tau$ using the following first-order Lie-Trotter method [16]:

$$
\phi(x, y, t+\tau)=\mathcal{S}_{A}^{\tau} \circ \mathcal{S}_{B}^{\tau} \circ \mathcal{S}_{C}^{\tau} \phi(x, y, t) .
$$

where $\delta_{A}^{\tau}$, $\mathcal{\delta}_{B}^{\tau}$, and $\delta_{C}^{\tau}$ are the exact solution operators associated with the nonlinear equation

$$
\phi_{t}=-(\Delta+1)^{2} \phi
$$

the heat equation

$$
\phi_{t}=-f(\phi),
$$

and the Lagrange multiplier equation

$$
\phi_{t}=\frac{1}{|\Omega|} \int_{\Omega}(\phi+f(\phi)) \mathrm{d} \mathbf{x},
$$

respectively. Higher order splitting schemes can be found in $[18,19]$.

Before proceeding to present a fast explicit operator splitting method for (4), the exact solution operators $\mathcal{S}_{A}^{\tau}, \mathcal{S}_{B}^{\tau}$, and $\delta_{C}^{\tau}$ are first replaced by their numerical approximations $\mathcal{S}_{A}^{\tau, h}, \mathcal{S}_{B}^{\tau, h}$, and $\mathcal{S}_{C}^{\tau, h}$, respectively.

Step $1\left(\mathcal{S}_{A}^{\tau} \longrightarrow \mathcal{S}_{A}^{\tau, h}\right)$. We first consider the fully discrete scheme for (11). The following spatial grid is introduced:

$$
\Omega_{h}^{\text {per }}=\left\{\left(x_{i}, y_{j}\right)=(a+i h, a+j h), \quad 0 \leq i, j \leq N-1\right\},
$$

for the periodic condition, where $h=(b-a) / N$. Let $u_{j, k}^{k}=u\left(x_{i}, y_{j}, k \tau\right)$, where $\tau=T / K$ is the time step and $M$ is the total number of time steps.

Based on the theory of spectral method [20], the solution to equation (11) derived with periodic boundary conditions can be represented by the following band-limited Fourier series: 


$$
\begin{aligned}
\mathscr{F}_{N}^{-1}[\widetilde{\phi}(t)](x, y) & :=\left(P_{N} \phi\right)(x, y, t) \\
& =\sum_{p=-N / 2}^{N / 2} \sum_{q=-N / 2}^{N / 2} \widetilde{\phi}_{p, q}(t) \varphi_{p, q}(x, y),
\end{aligned}
$$

where

$$
\varphi_{p, q}=\exp \left(i \frac{2 p \pi(x-a)}{b-a}+i \frac{2 q \pi(y-a)}{b-a}\right),
$$

and by using the following Fourier expansion, the fast Fourier transform (FFT) may be used to calculate the Fourier coefficients $\left\{\widetilde{\phi}_{p, q}(t)\right\}$ from the discrete function values $\left\{\phi_{i, j}(t)\right\}$ :

$$
\begin{aligned}
\mathscr{F}_{N}[\phi(t)](p, q):= & \widetilde{\phi}_{p, q}(t)=\frac{h^{2}}{C_{p}^{\mathrm{per}} C_{q}^{\mathrm{per}}(b-a)^{2}} \sum_{j=0}^{N-1} \sum_{k=0}^{N-1} \phi_{i, j} \\
& \cdot(t) \varphi_{p, q}\left(x_{i}, y_{j}\right),
\end{aligned}
$$

and $C_{p}^{\text {per }}$ and $C_{q}^{\text {per }}$ are, respectively, defined as

$$
C_{r}^{\mathrm{per}}= \begin{cases}2, & |r|=\frac{N}{2}, \\ 1, & |r|<\frac{N}{2} .\end{cases}
$$

Substituting (15) into (11), we obtain the following system of ordinary differential equations for the discrete ( $p q)$-th Fourier coefficients:

$$
\frac{\mathrm{d} \widetilde{\phi}_{p, q}(t)}{\mathrm{d} t}=-\left(\lambda_{p, q}+1\right)^{2} \widetilde{\phi}_{p, q}(t),
$$

where

$$
\lambda_{p, q}=-\left(\left(\frac{2 p \pi}{b-a}\right)^{2}+\left(\frac{2 q \pi}{b-a}\right)^{2}\right), \quad p, q=0, \pm 1, \pm 2, \ldots, \pm \frac{N}{2} .
$$

The above equation is solved analytically by the variable separation method with an initial condition $\widehat{\phi}_{p q}^{k}$, and then the solution $\widehat{\phi}_{p q}^{k+1}$ is obtained as follows:

$$
\widetilde{\phi}_{p, q}^{k+1}=\exp \left(-\tau\left(\lambda_{p, q}+1\right)^{2}\right) \tilde{\phi}_{p, q}^{k} .
$$

Hence, we have

$$
\phi^{k+1}=\mathscr{F}_{N}^{-1}\left\{\exp \left(-\tau\left(\lambda_{p, q}+1\right)^{2}\right) \mathscr{F}_{N}\left[\phi^{k}\right](p, q)\right\} .
$$

The errors between $P_{N} \phi$ and $\phi$ can be measured in Sobolev spaces. For this purpose, we denote by $H_{p}^{s}(\Omega)$ the subspace of $H^{s}(\Omega)$, which consists of functions with derivatives of order up to $s-1$ being periodic on $\Omega$. The main approximation result is stated below.
Theorem 1 (see [20]). For any $\phi \in H_{p}^{s}(\Omega)$ and $s \geq 0$, it holds that

$$
\left\|P_{N} \phi-\phi\right\| \leq C N^{-s}|\phi|_{s}
$$

This theorem indicates that the projection $P_{N} \phi$ is the best approximation of $\phi$ in all Sobolev spaces $H_{p}^{s}(\Omega)$. For further references about the spectral method, we refer to [20].

Step $2\left(\mathcal{S}_{B}^{\tau} \longrightarrow \mathcal{S}_{B}^{\tau, h}\right)$. Now we propose a first-order difference scheme for solving equation (12) in $\left[t_{k}, t_{k+1}\right]$. Note that if $|\phi| \neq 0$, equation (12) can be rewritten as

$$
\frac{\phi_{t}}{\phi}=-\left(\phi^{2}-\eta \phi-\epsilon\right) .
$$

Taking the integral from $t_{k}$ to $t_{k+1}$ on both sides of the above equation with respect to $t$, we have

$$
\ln \phi^{k+1}-\ln \phi^{k}=-\int_{t_{k}}^{t_{k+1}}\left(\phi^{2}-\eta \phi-\epsilon\right) \mathrm{d} t .
$$

Note that $\int_{t_{k}}^{t_{k+1}}\left(\phi^{2}-\eta \phi-\epsilon\right) \mathrm{d} t=\tau\left(\left(\phi^{k}\right)^{2}-\eta \phi^{k}-\epsilon\right)+$ $O\left(\tau^{2}\right)$, and then we obtain a first-order explicit finite difference scheme for (12)

$$
\phi^{k+1}=\phi^{k} \exp \left(-\tau\left(\left(\phi^{k}\right)^{2}-\eta \phi^{k}-\epsilon\right)\right) .
$$

It is clear that if $|\phi|=0$, the above formula is also derived.

Step $3\left(\mathcal{S}_{C}^{\tau} \longrightarrow \mathcal{S}_{C}^{\tau, h}\right)$. Equation (12) involves solving a nonlocal Lagrange multiplier equation. For convenience, we denote $\beta(\phi)=\int_{\Omega}(\phi+f(\phi)) \mathrm{d} \mathbf{x}$. Then, we employ the backward difference method for equation (13) in $\left[t_{k}, t_{k+1}\right]$ with an initial condition $\phi^{k}$; this gives

$$
\frac{\phi^{k+1}-\phi^{k}}{\tau}=\frac{1}{|\Omega|} \beta\left(\phi^{k+1}\right) .
$$

Summing both sides of equation (27) over $i$ and $j$, and by the property of mass conservation $h^{2} \sum_{i, j=0}^{N-1} \phi_{i j}^{0}=h^{2} \sum_{i, j=0}^{N-1} \phi_{i j}^{k+1}$, we obtain

$$
\beta\left(\phi^{k+1}\right)=\frac{h^{2}}{\tau}\left(\sum_{i, j=0}^{N-1} \phi_{i j}^{0}-\sum_{i, j=0}^{N-1} \phi_{i j}^{k}\right) .
$$

A similar idea has also been mentioned in [21].

Combining (27) and (28), a first-order explicit finite difference scheme for (13) is obtained:

$$
\phi^{k+1}=\phi^{k}+\frac{h^{2}}{|\Omega|}\left(\sum_{i, j=0}^{N-1} \phi_{i j}^{0}-\sum_{i, j=0}^{N-1} \phi_{i j}^{k}\right) .
$$

Then, based on equations (10), (22), (26), and (29), we obtain the following fast explicit operator splitting schemes: 
Table 1: Convergence rate with $h=1 / 2$ and different $M$ for Problem 1 at $T=1$.

\begin{tabular}{|c|c|c|c|c|c|c|}
\hline & $M=$ & 10 & 20 & 40 & 80 & 160 \\
\hline & $\operatorname{Err}_{L^{\infty}}$ & $8.14 e-5$ & $1.78 e-5$ & $4.18 e-6$ & $1.01 e-6$ & $2.49 e-7$ \\
\hline$\epsilon=0.05$ & Rate $_{L^{\infty}}$ & - & 2.19 & 2.09 & 2.05 & 2.02 \\
\hline \multirow[t]{3}{*}{$\eta=0.05$} & $\operatorname{Err}_{L^{2}}$ & $1.30 e-3$ & $2.95 e-4$ & $7.04 e-5$ & $1.72 e-5$ & $4.25 e-6$ \\
\hline & Rate $_{L^{2}}$ & - & 2.14 & 2.07 & 2.03 & 2.02 \\
\hline & $\operatorname{Err}_{L^{\infty}}^{L}$ & $4.61 e-3$ & $1.10 e-3$ & $2.66 e-4$ & $6.52 e-5$ & $1.62 e-5$ \\
\hline$\epsilon=0.25$ & Rate $_{L^{\infty}}$ & - & 2.07 & 2.05 & 2.03 & 2.01 \\
\hline \multirow[t]{3}{*}{$\eta=2$} & $\operatorname{Err}_{L^{2}}$ & $3.69 e-2$ & $9.16 e-3$ & $2.27 e-3$ & $5.63 e-4$ & $1.40 e-4$ \\
\hline & Rate $_{L^{2}}$ & - & 2.01 & 2.01 & 2.01 & 2.01 \\
\hline & $\operatorname{Err}_{L^{\infty}}^{L}$ & $1.25 e-4$ & $3.30 e-5$ & $8.43 e-6$ & $2.13 e-6$ & $5.34 e-7$ \\
\hline & Rate $_{L^{\infty}}$ & - & 1.92 & 1.97 & 1.99 & 1.99 \\
\hline \multirow{2}{*}{$\eta=0.5$} & $\operatorname{Err}_{L^{2}}$ & $1.78 e-3$ & $4.23 e-4$ & $1.03 e-4$ & $2.54 e-5$ & $6.32 e-6$ \\
\hline & Rate $_{L^{2}}$ & - & 2.08 & 2.04 & 2.02 & 2.01 \\
\hline
\end{tabular}

$$
\left\{\begin{array}{l}
\phi^{k+1,(1)}=\mathscr{F}_{N}\left\{\exp \left(-\tau\left(\lambda_{p, q}+1\right)^{2}\right) \mathscr{F}_{N}^{-1}\left[\phi^{k}\right](p, q)\right\} \\
\phi^{k+1,(2)}=\phi^{k+1,(1)} \exp \left(-\tau\left(\left(\phi^{k}\right)^{2}-\eta \phi^{k}-\epsilon\right)\right) \\
\phi^{k+1}=\phi^{k+1,(2)}+\frac{h^{2}}{|\Omega|}\left(\sum_{i, j=0}^{N-1} \phi_{i j}^{0}-\sum_{i, j=0}^{N-1} \phi_{i j}^{k,(2)}\right)
\end{array}\right.
$$

The derivation of algorithm (30) shows that the temporal truncation error has the form $\left(C_{1} \tau+C_{2} \tau^{2}\right)$. Based on this observation, we can use the following Richardson's extrapolation technique:

$$
\phi_{w}^{K}=2 \phi^{2 K}-\phi^{K}
$$

to eliminate the leading term $O(\tau)$, where $\phi^{K}$ and $\phi^{2 K}$ are the numerical solutions at the final time level calculated using time step sizes $\tau=T / K$ and $\tau=T / 2 K$, respectively. Thus, we obtain a high-order numerical solution $\phi_{w}^{K}$ with secondorder temporal accuracy.

In addition, we define the discrete inner product

$$
(f, g):=h^{2} \sum_{i, j=0}^{N-1} f_{i, j} g_{i, j}
$$

and discrete $\|\cdot\|_{\infty}$ norm and $\|\cdot\|_{2}$ norm

$$
\begin{aligned}
\|f\|_{\infty} & =\max _{0 \leq i, j \leq N-1}\left|f_{i, j}\right|, \\
\|f\|_{2} & =\sqrt{(f, f)} .
\end{aligned}
$$

Theorem 2. Scheme (30) satisfies the discrete mass conservation law

$$
M^{k}=M^{0}, \quad k=1,2, \ldots, K
$$

where $M^{k}=h^{2} \phi_{i, j}^{k}$

Proof. Taking discrete inner product of the third equation of (30) with 1 , summing over $i$ and $j$, we have

$$
\begin{aligned}
\left(\phi^{k+1}, 1\right) & =\left(\phi^{k+1,(2)}, 1\right)+\left(\frac{h^{2}}{|\Omega|}\left(\sum_{i, j=0}^{N-1} \phi_{i j}^{0}-\sum_{i, j=0}^{N-1} \phi_{i j}^{k,(2)}\right), 1\right) \\
& =\left(\phi^{0}, 1\right) .
\end{aligned}
$$

This completes the proof.

\section{Numerical Experiments}

In this section, three numerical experiments are carried out to study the accuracy and efficiency of the proposed method. The $L^{\infty}$-norm error (denoted as $\operatorname{Err}_{L^{\infty}}$ ) and the $L^{2}$-norm error (denoted as $\operatorname{Err}_{L^{2}}$ ) of the numerical solutions are computed, respectively, by the following formulas:

$$
\begin{gathered}
\operatorname{Err}_{L^{\infty}}=\left\|\phi^{M+1}-\phi(x, y, T)\right\|_{\infty}, \\
\operatorname{Err}_{L^{2}}=\left\|\phi^{M+1}-\phi(x, y, T)\right\|_{2} .
\end{gathered}
$$

Problem 1 (convergence test). In order to verify the temporal numerical accuracy, we consider an example on $[0,64]^{2} \times(0,1]$ with various $\epsilon$ and $\eta$, and the initial condition is as follows:

$$
\phi_{0}(x, y)=\sin \left(\frac{8 \pi}{128} x\right) \cos \left(\frac{8 \pi}{128} y\right) .
$$

We use $h=1 / 2$, so the spatial discretization errors are negligible compared with the time discretization error. The computational results at $T=1$ for various $\epsilon$ and $\eta$ are presented in Table 1. One may see that the convergence rate is close to 2 for all cases, which coincides with what we expected.

Problem 2 (effects of $\epsilon$ and $\eta$ on energy stability and pattern formation). Various related works have been proposed for the Swift-Hohenberg equation, and most of them considered the case of $\eta=0$ except $[9,10]$. In order to show the effects of $\epsilon$ and $\eta$ on pattern formation, we use a benchmark simulation for the conservative Swift-Hohenberg equation, 


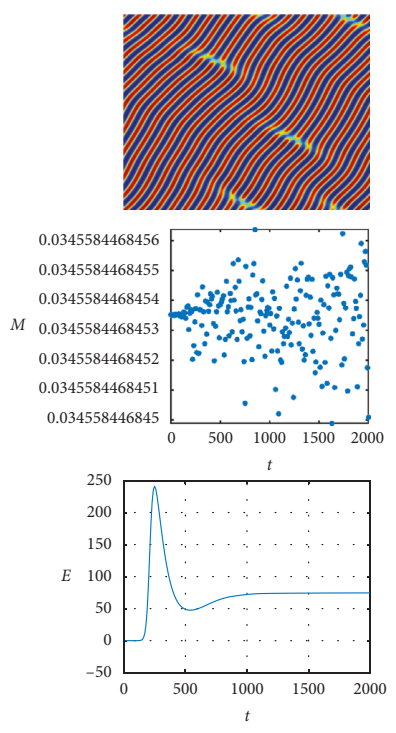

(a)

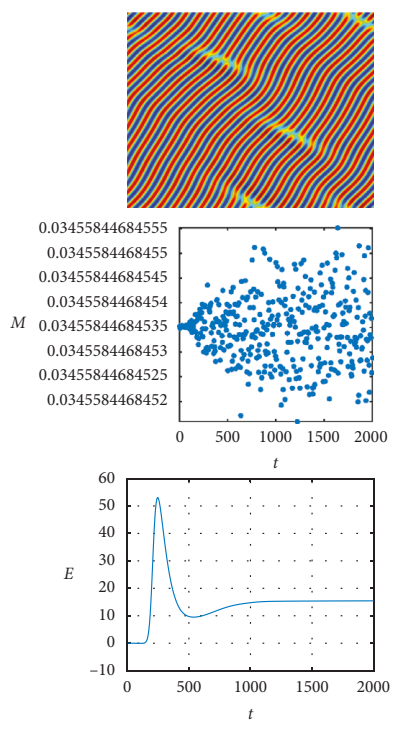

(b)
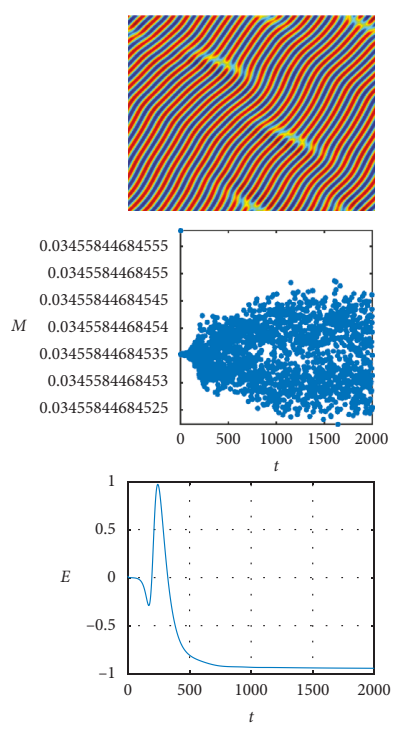

(c)

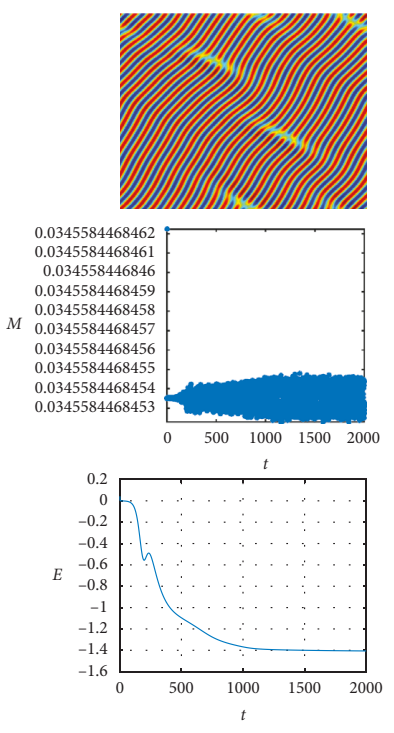

(d)
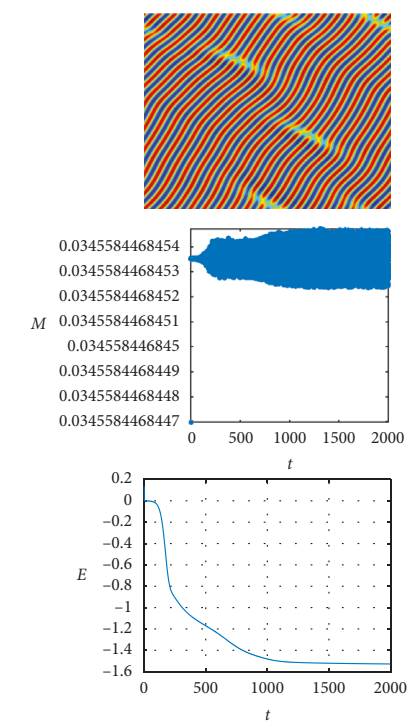

(e)

Figure 1: $\phi(x, y, 2000)$ and the curves of mass $M$ and energy $E$ with different time steps $\tau$ for Problem 2 : (a) $\tau=10$, (b) $\tau=5$, (c) $\tau=1$, (d) $\tau=0.5$, and (e) $\tau=0.1$.

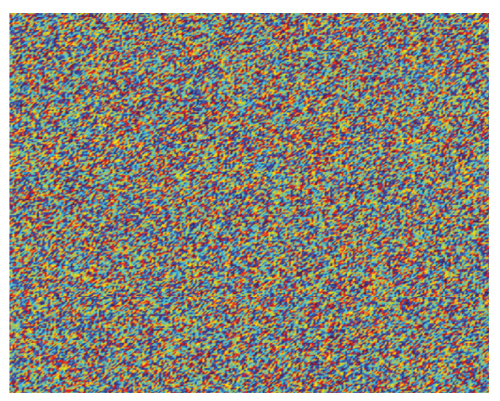

(a)

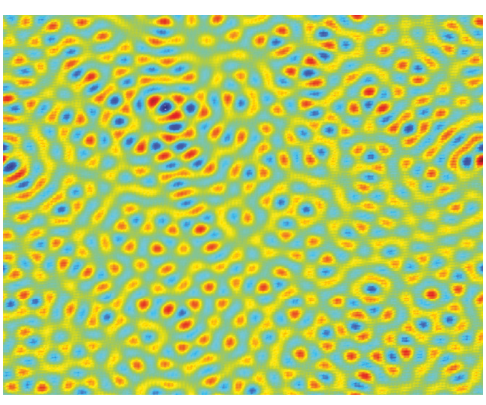

(b)

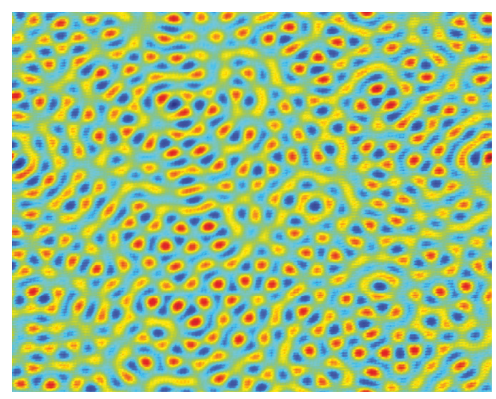

(c)

Figure 2: Continued. 


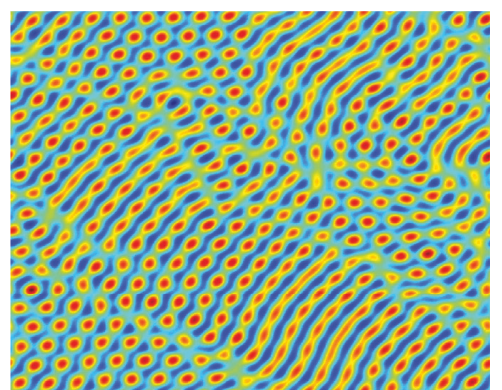

(d)

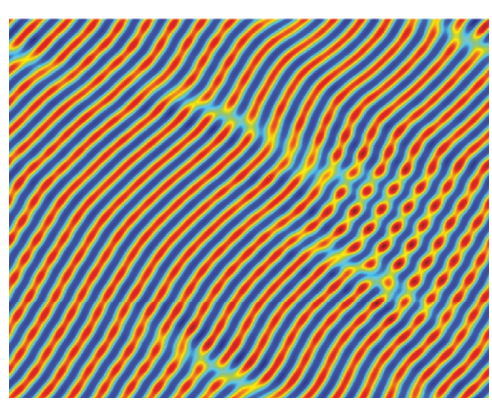

(e)

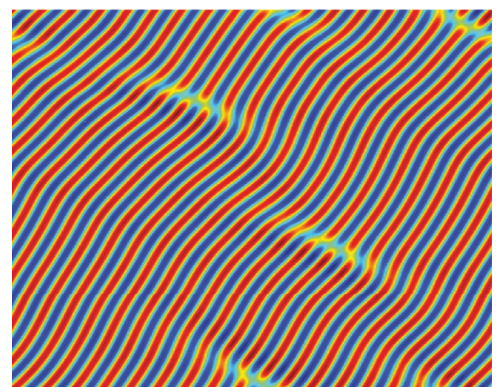

(f)

Figure 2: Snapshots of $\phi$ at different time steps for Problem 2: (a) $\phi(x, y, 0)$, (b) $\phi(x, y, 100),(\mathrm{c}) \phi(x, y, 200),(\mathrm{d}) \phi(x, y, 600)$, (e) $\phi(x, y, 1000)$, and (f) $\phi(x, y, 2000)$.
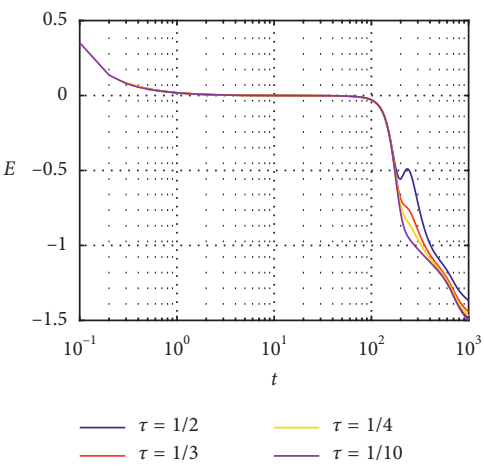

(a)

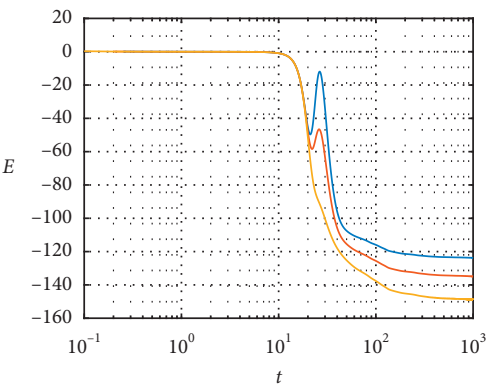

$\tau \tau=1 / 4$

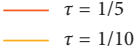

(d)
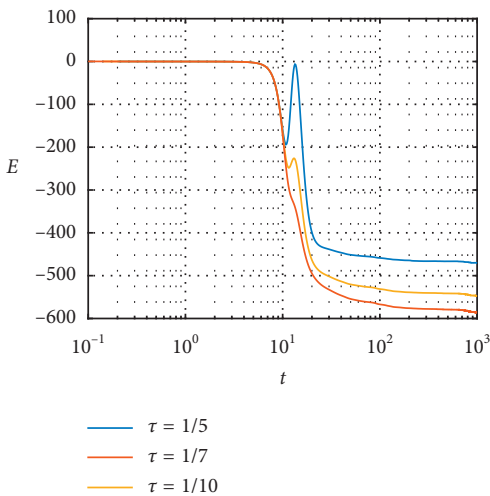

(g)

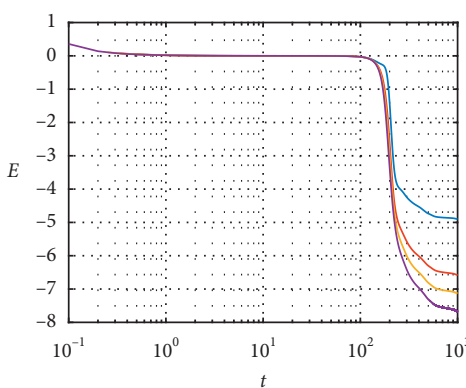

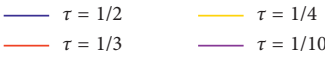

(b)
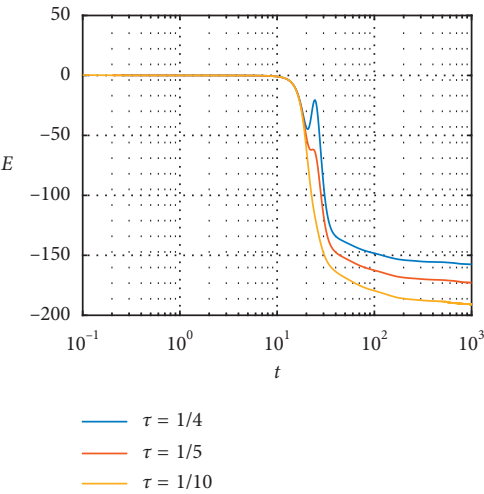

(e)
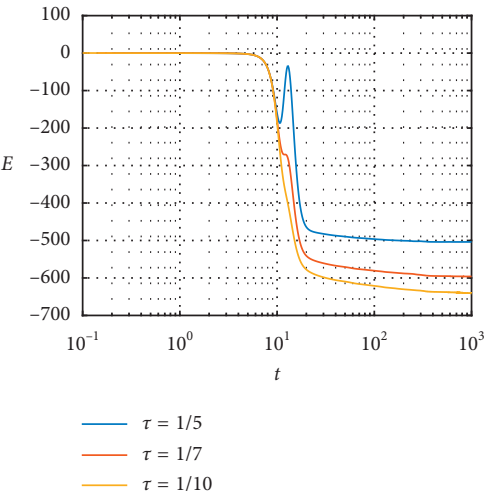

(h)

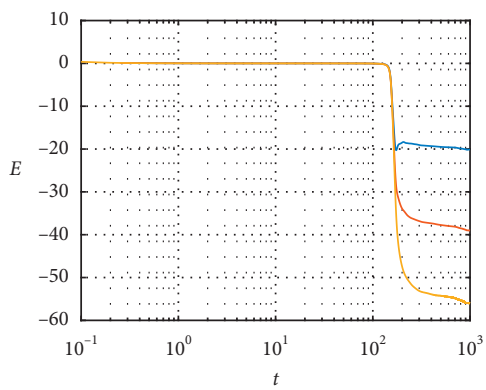

$-\tau=1 / 3$

$\tau-\tau=1 / 4$

(c)
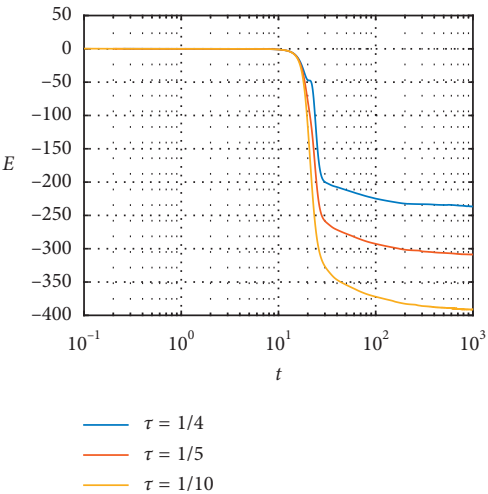

(f)

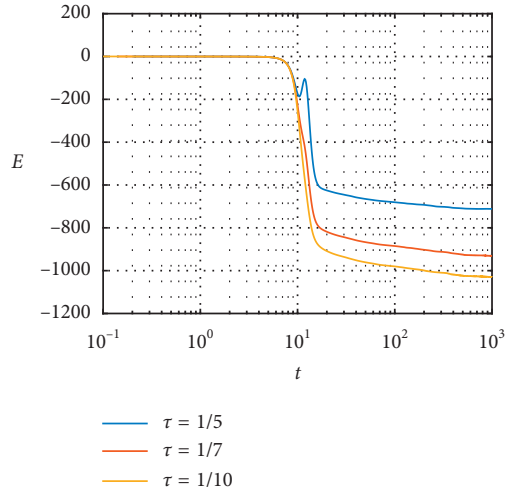

(i)

Figure 3: The curves of energy $E$ with different $\epsilon, \eta$, and time steps $\tau$ for Problem 2: (a) $\epsilon=0.025, \eta=0.1$, (b) $\epsilon=0.025, \eta=0.5$, (c) $\epsilon=0.025, \eta=0.99$, (d) $\epsilon=0.25, \eta=0.1$, (e) $\epsilon=0.25, \eta=0.5$, (f) $\epsilon=0.25, \eta=0.99$, (g) $\epsilon=0.5, \eta=0.1$, (h) $\epsilon=0.5, \eta=0.5$, and (i) $\epsilon=0.5, \eta=0.99$. 


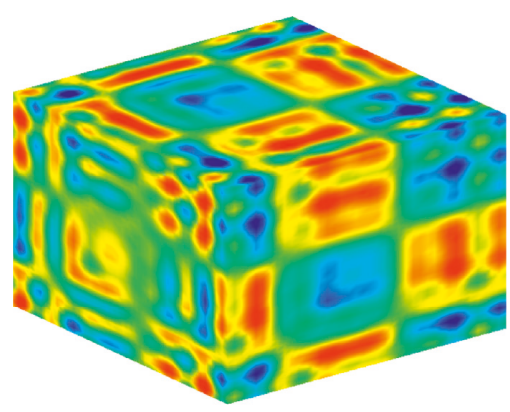

(a)

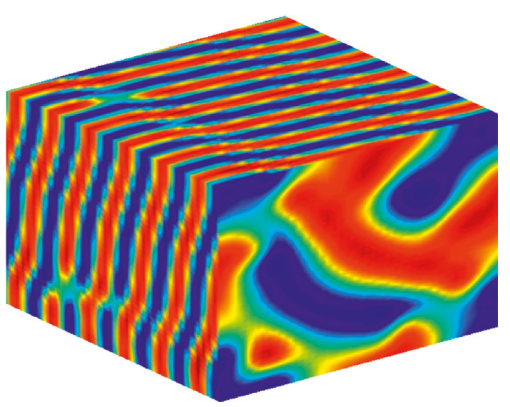

(d)

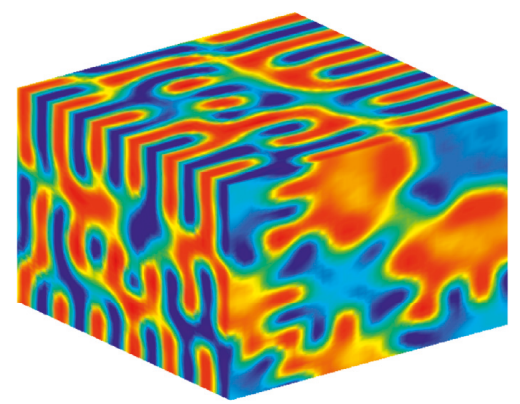

(b)

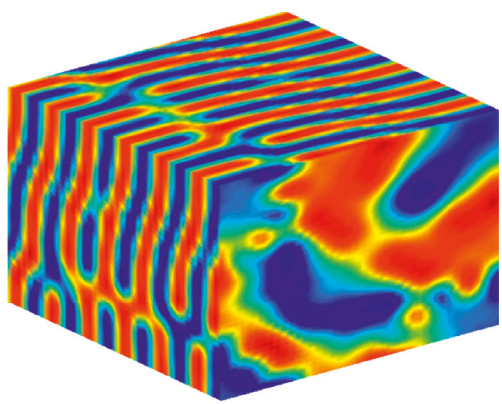

(c)

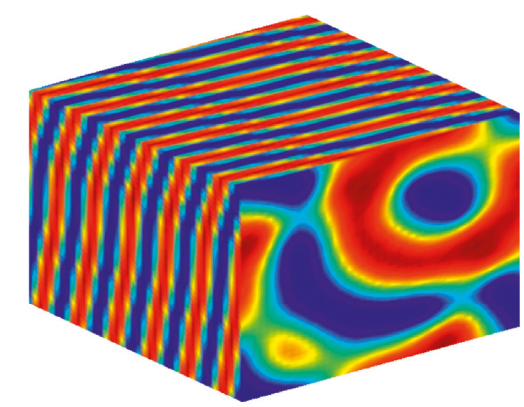

(e)

Figure 4: Snapshots of $\phi$ at different time periods for Problem 3: (a) $\phi(x, y, 10),($ b) $\phi(x, y, 100),($ c) $\phi(x, y, 300),(d) \phi(x, y, 400)$, and (e) $\phi(x, y, 1000)$.

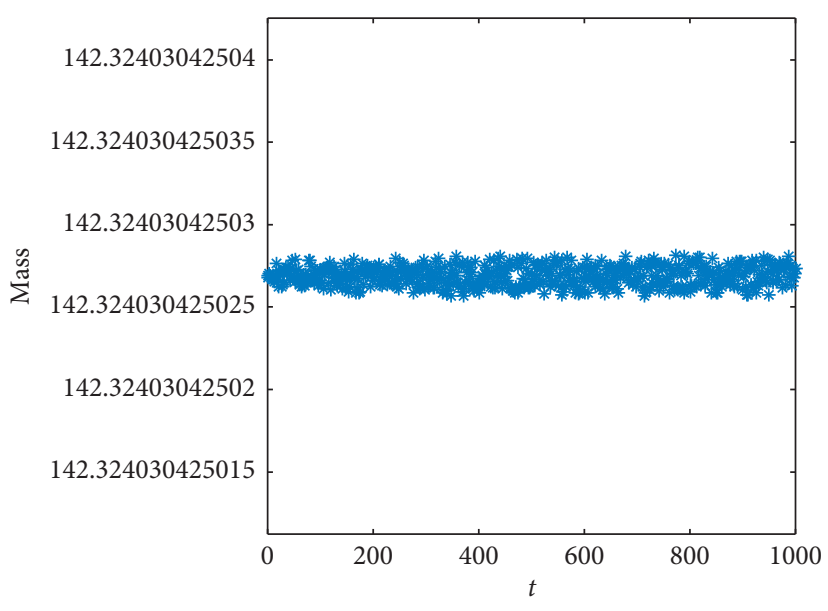

(a)

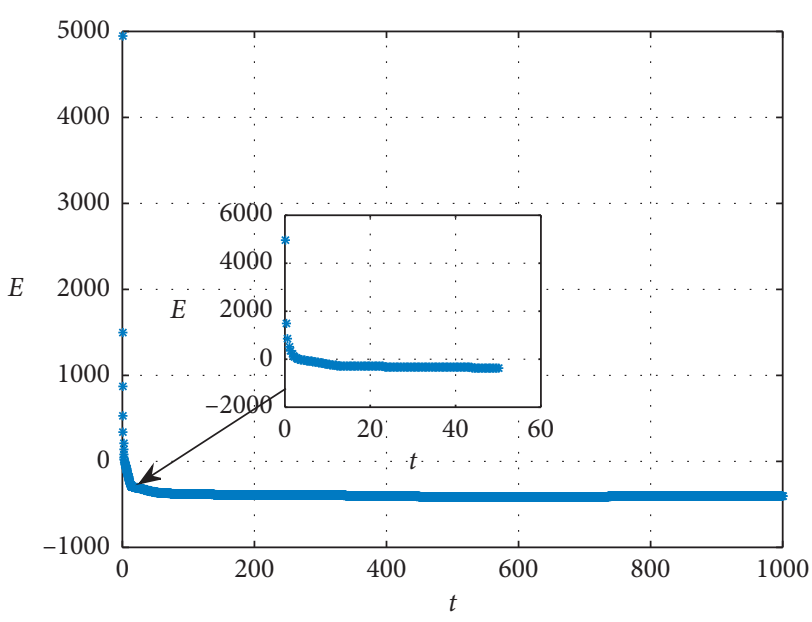

(b)

Figure 5: The curves of (a) mass $M$ and (b) energy $E$ for Problem 3.

i.e., the phase transition behaviors. We set the $2 \mathrm{D}$ computational domain as $[0,128]^{2}$ and discretize it by using $h=1$. The initial condition reads as

$$
\phi_{0}(x, y)=0.07(1+\operatorname{rand}(x, y)),
$$

where $\operatorname{rand}(x, y)$ is a random number in the range of $[-1,1]$.

We first perform a numerical experiment with $\epsilon=0.025$ and $\eta=0.1$ for the stability study of the proposed method. Figure 1 shows the $x-y-\phi$ plots at $T=2000$ with different time steps, and the corresponding curves of mass $M$ and energy $E$ are also presented. These results suggest that the proposed method satisfies mass conservation and is unconditionally stable. The only drawback is that the method is conditionally energy stable.

Secondly, with the same parameters and $\tau=0.1$, we present the snapshots of phase transition behaviors of the density field $\phi(x, y, t)$ at different computational time steps in Figure 2.

Finally, the relationship between energy stability and the parameters $\epsilon$ and $\eta$ is examined. Figure 3 shows the evolution of the energies using different $\epsilon, \eta$, and time steps. The results in Figure 3 suggest that the proposed method is conditionally energy stable. We can see from this figure that 
the energy $E(\phi)$ is mainly affected by the value of $\epsilon$ rather than $\eta$, and small time step $\tau$ is needed to keep the energy law (7) for large $\epsilon$.

Problem 3 (the conservative Swift-Hohenberg equation in $3 \mathrm{D})$. We consider the evolution of $\phi$ in $3 \mathrm{D}$ space using the proposed method. The initial condition on $[0,40]^{3}$ is

$$
\phi_{0}(x, y, z)=\sin \left(\frac{8 \pi x}{128}\right) \cos \left(\frac{8 \pi y}{128}\right) \cos \left(\frac{8 \pi z}{128}\right) .
$$

We take the simulation parameters

$$
\begin{aligned}
& T=1000, \\
& \epsilon=0.25, \\
& \eta=0.05, \\
& h=1, \\
& \tau=\frac{1}{3} .
\end{aligned}
$$

In Figure 4, we display the evolution of the phase field at different time periods. From the results, it can be seen that our method is applicable to 3D conservative SwiftHohenberg equation. Moreover, the curves of mass $M$ and energy $E$ are also presented in Figure 5.

\section{Conclusions}

In this paper, we developed a second-order, linear, and mass conservation algorithm for solving the nonlocal Swift-Hohenberg equation by combining the operator splitting technique with the Fourier spectral method. A variety of numerical experiments were presented to demonstrate the stability and the accuracy of the developed method. In particular, it is applicable to various problems with a mass conservation constraint.

\section{Data Availability}

The data used to support the findings of this study are included within the article.

\section{Conflicts of Interest}

The authors declare that they have no conflicts of interest.

\section{Acknowledgments}

This work was supported by the Natural Science Foundation of Xinjiang Province (No. 2016D01C071).

\section{References}

[1] J. Swift and P. C. Hohenberg, "Hydrodynamic fluctuations at the convective instability," Physical Review A, vol. 15, no. 1, pp. 319-328, 1977.
[2] A. Hutt and F. M. Atay, "Analysis of nonlocal neural fields for both general and gamma-distributed connectivities," Physica D: Nonlinear Phenomena, vol. 203, no. 1-2, pp. 30-54, 2005.

[3] A. Hutt, A. Longtin, and L. Schimansky-Geier, "Additive noise-induced Turing transitions in spatial systems with application to neural fields and the Swift-Hohenberg equation," Physica D: Nonlinear Phenomena, vol. 237, no. 6, pp. 755-773, 2008.

[4] M. Brassel and E. Bretin, "A modified phase field approximation for mean curvature flow with conservation of the volume," Mathematical Methods in the Applied Sciences, vol. 34, no. 10, pp. 1157-1180, 2011.

[5] C. I. Christov, J. Pontes, D. Walgraef, and M. G. Velarde, "Implicit time splitting for fourth-order parabolic equations," Computer Methods in Applied Mechanics and Engineering, vol. 148, no. 3-4, pp. 209-224, 1997.

[6] C. I. Christov and J. Pontes, "Numerical scheme for SwiftHohenberg equation with strict implementation of lyapunov functional," Mathematical and Computer Modelling, vol. 35, no. 1-2, pp. 87-99, 2002.

[7] M. Cheng and J. A. Warren, "An efficient algorithm for solving the phase field crystal model," Journal of Computational Physics, vol. 227, no. 12, pp. 6241-6248, 2008.

[8] M. Elsey and B. Wirth, "A simple and efficient scheme for phase field crystal simulation," ESAIM: Mathematical Modelling and Numerical Analysis, vol. 47, no. 5, pp. 1413-1432, 2013.

[9] H. Gomez and X. Nogueira, "A new space-time discretization for the Swift-Hohenberg equation that strictly respects the Lyapunov functional," Communications in Nonlinear Science and Numerical Simulation, vol. 17, no. 12, pp. 4930-4946, 2012.

[10] M. Dehghan and M. Abbaszadeh, "The meshless local collocation method for solving multi-dimensional Cahn-Hilliard, Swift-Hohenberg and phase field crystal equations," Engineering Analysis with Boundary Elements, vol. 78, pp. 49-64, 2017.

[11] H. G. Lee, "A semi-analytical Fourier spectral method for the Swift-Hohenberg equation," Computers \& Mathematics with Applications, vol. 74, no. 8, pp. 1885-1896, 2017.

[12] J. Su, W. W. Fang, Q. Yu, and Y. B. Li, "Numerical simulation of Swift-Hohenberg equation by the fourth-order compact scheme," Computational and Applied Mathematics, vol. 38, no. 2, pp. 1-15, 2019.

[13] Z. Weng, S. Zhai, and X. Feng, "Analysis of the operator splitting scheme for the Cahn-Hilliard equation with a viscosity term," Numerical Methods for Partial Differential Equations, vol. 35, no. 6, pp. 1949-1970, 2019.

[14] S. Zhai, L. Wu, J. Wang, and Z. Weng, "Numerical approximation of the fractional Cahn-Hilliard equation by operator splitting method," Numerical Algorithms, 2019.

[15] J. Zhang and X. Yang, "Numerical approximations for a new $L^{2}$-gradient flow based Phase field crystal model with precise nonlocal mass conservation," Computer Physics Communications, vol. 243, pp. 51-67, 2019.

[16] G. Strang, "On the construction and comparison of difference schemes," SIAM Journal on Numerical Analysis, vol. 5, no. 3, pp. 506-517, 1968.

[17] S. Zhai, D. Wang, Z. Weng, and X. Zhao, "Error analysis and numerical simulations of Strang splitting method for space fractional nonlinear Schrödinger equation," Journal of Scientific Computing, vol. 81, no. 2, pp. 965-989, 2019.

[18] S. Blanes and P. C. Moan, "Practical symplectic partitioned Runge-Kutta and Runge-Kutta-Nyström methods," Journal of 
Computational and Applied Mathematics, vol. 142, no. 2, pp. 313-330, 2002.

[19] P. Csomós, I. Faragó, and Á. Havasi, "Weighted sequential splittings and their analysis," Computers \& Mathematics with Applications, vol. 50, no. 7, pp. 1017-1031, 2005.

[20] J. Shen, T. Tang, and L. L. Wang, Spectral Methods Algorithms: Analyses and Applications, Springer, Berlin, Germany, 1st edition, 2010.

[21] J. Kim, S. Lee, and Y. Choi, "A conservative Allen-Cahn equation with a space-time dependent Lagrange multiplier," International Journal of Engineering Science, vol. 84, pp. 11-17, 2014. 


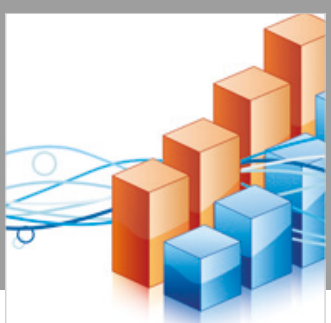

Advances in

Operations Research

\section{-n-m}
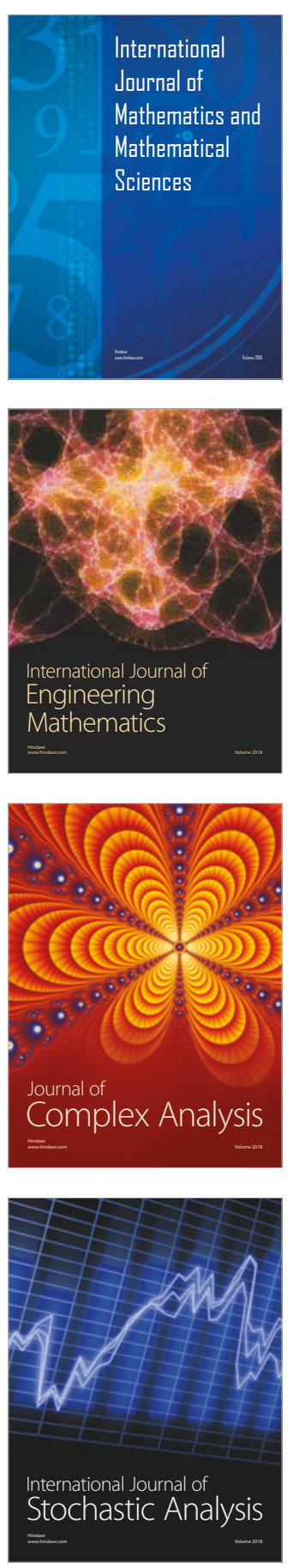
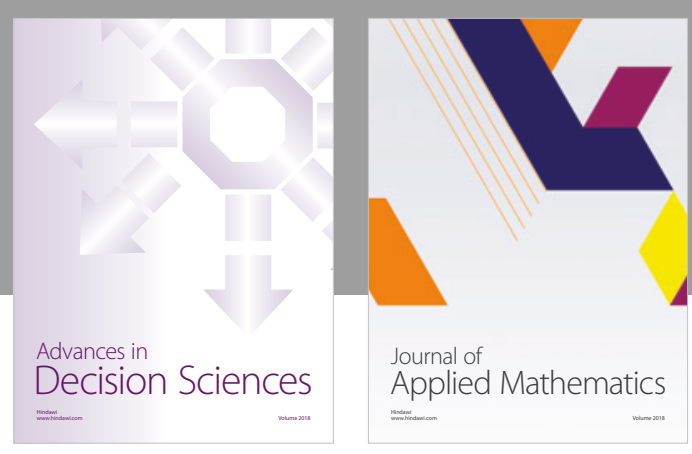

Journal of

Applied Mathematics
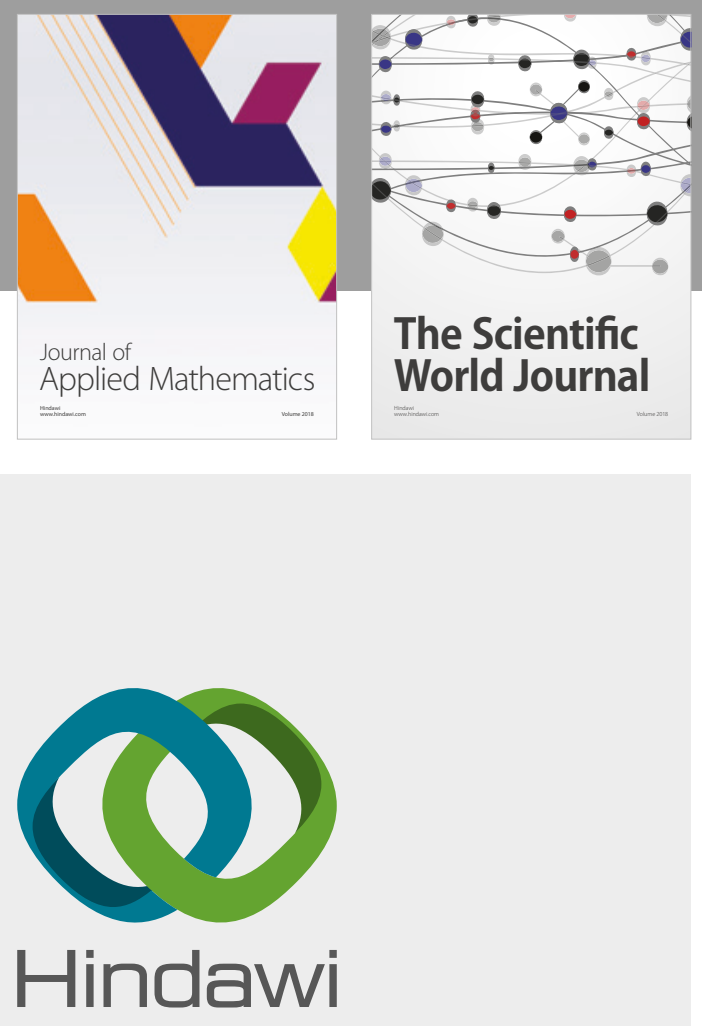

Submit your manuscripts at

www.hindawi.com

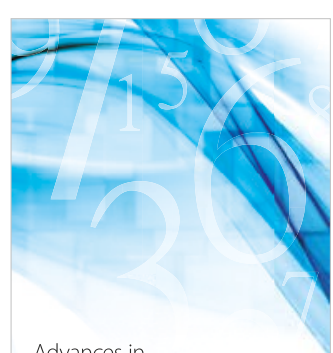

Advances in
Numerical Analysis
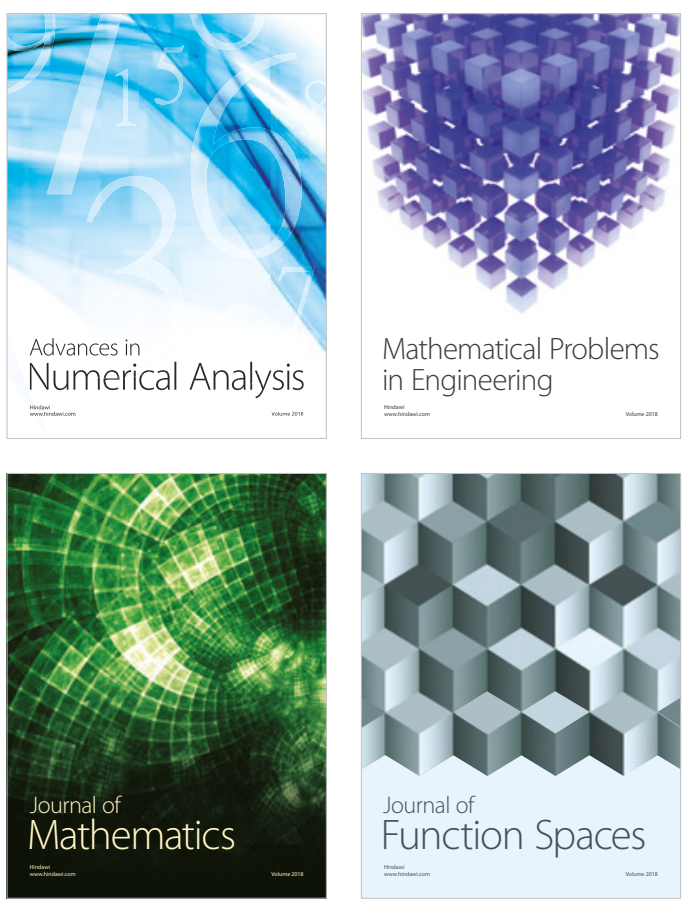

Mathematical Problems in Engineering

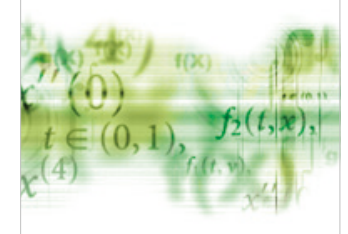

International Journal of

Differential Equations

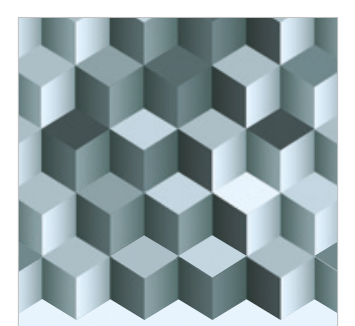

Journal of

Function Spaces

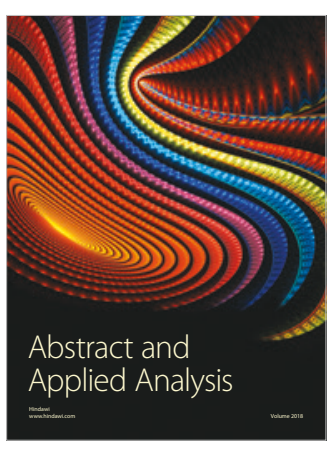

The Scientific

World Journal

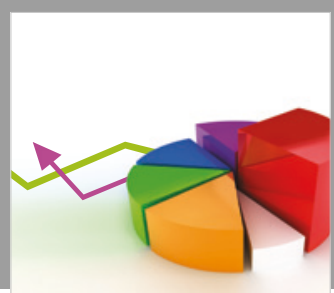

Journal of

Probability and Statistics
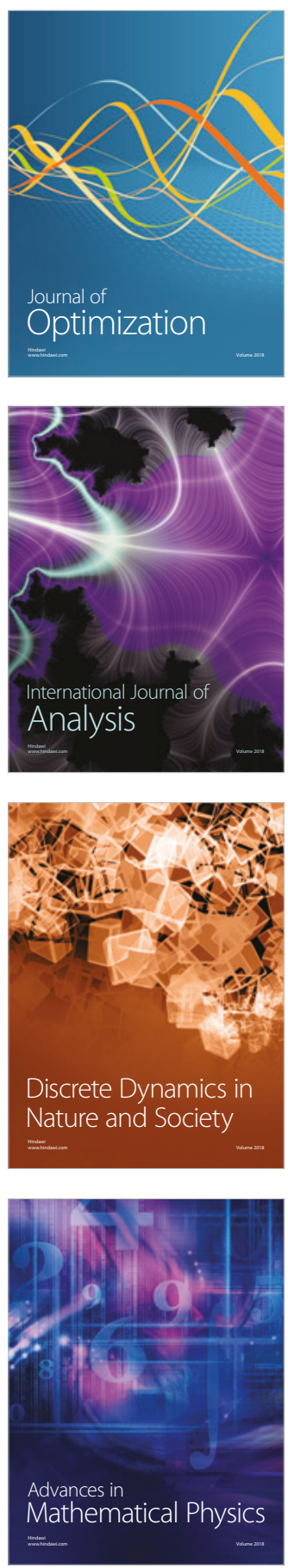